\title{
DIRECT VISUAL INTERNAL URETHROTOMY (DVIU) FOR UNTREATED BULBAR URETHRAL STRICTURES. A SOLUTION?
}

\begin{tabular}{l|l}
$\begin{array}{l}\text { Dr Abhiyutthan } \\
\text { Singh Jadaon* }\end{array}$ & $\begin{array}{l}\text { Mch Urology Abhiyutthanum Hospital Kota, Rajasthan, India. } \\
{ }^{*} \text { Correspondiong Author }\end{array}$ \\
\hline $\begin{array}{l}\text { Dr Chitra } \\
\text { Champawat }\end{array}$ & MS Obgy Abhiyutthanum hospital Kota, Rajasthan, India. \\
\hline $\begin{array}{l}\text { Dr Chandra Pal } \\
\text { Singh }\end{array}$ & MS General Surgery Kota Heart Hospital Kota, Rajasthan, India.
\end{tabular}

ABSTRACT

Introduction :Urethral stricture diseases have been treated with numerous approaches. Though open urethroplasty is considered a one-time solution [1], The direct visual internal urethrotomy (DVIU) is still considered an alternative approach in the stepladder of treatment $[2,3]$. Here we report our experience at a tertiary care hospital with DVIU in a homogeneous series of patients with bulbar urethral stricture who underwent strict follow-up and present a multivariable analysis of the results to identify significant predictors of treatment failure. We wish to offer new insights into DVIU. Materials and method: We performed a retrospective analysis of patients who underwent internal urethrotomy. Patients who underwent DVIU for untreated bulbar urethral strictures with minimum follow-up of 12 months were included. Patients with traumatic stricture and stricture length $>4 \mathrm{~cm}$ were excluded.The primary outcome was treatment failure. Multivariable Cox regression analyses by Stata v.12.0 were used to test the association between predictors:- Stricture etiology, stenosis length, preoperative maximum flow $[\mathrm{pQmax}]$ ) and treatment failure. Results: 215 patients were included. Median follow-up was 35 months. At 5-yr follow-up the failure-free survival rate was $54.4 \%$. On multivariable analysis pQmax was the only significant predictor of treatment failure. Conclusions: DVIU success rate for untreated bulbar urethral strictures was significantly associated with preoperative maximum flow rate. The patients with a pre- operative maximum flow lesser than 6 $\mathrm{ml} / \mathrm{s}$ have a low probability of success and may be considered for alternative treatments such as open urethroplasty, especially when affected by long urethral strictures.

\section{KEYWORDS : DVIU; Pqmax; Stricture.}

\section{INTRODUCTION}

Urethral stricture diseases have been treated with numerous approaches. Though Open urethroplasty is considered a onetime solution [1], The direct visual internal urethrotomy (DVIU) is still considered an alternative approach in the stepladder of treatment $[2,3]$

Results of DVIU has been poles apart in studies with some claiming it to have more than $80 \%$ success rate [4], with others on another end claiming them to be mere 8.3\%. [5]. However, the failure rate after initial DVIU is reported to be at least $50 \%$, and for the majority of patients this procedure should be considered as a temporizing measure until definitive reconstruction can be planned [6]. It's important to note that these negative conclusions often originate from nonhomogeneous series with poor follow-up [6-8].

Thus here we report our experience at a tertiary care hospital with DVIU in a homogeneous series of patients with bulbar urethral stricture who underwent strict follow-up, and present a multivariable analysis of the results to identify significant predictors of treatment failure. We wish to offer new insights into DVIU.

\section{MATERIALS AND METHOD}

Patient population and study design

We performed a retrospective descriptive analysis for a cohort of patients who underwent DVIU at our institute in the last 6 years. The institutional ethics committee approved the study. Informed consent was taken from all patients, explaining the surgical procedure and the complications. Patients were fully informed about the stricture recurrence rate that the current literature suggests for DVIU.

Inclusion criteria

Patients who underwent DVIU for untreated bulbar urethral strictures with minimum follow-up of 12 months.

\section{Exclusion criteria}

Patients with traumatic stricture and stricture length more than $4 \mathrm{~cm}$.

\section{Primary outcome}

Treatment failure was the primary outcome of the study, which was, defined as when patients underwent a new surgical procedure for stricture recurrence.

\section{Preoperative workup, variables included in study}

All patients underwent preoperative evaluation using urine culture, uroflowmetry, post-void residual (PVR), retrograde urethrogram (RUG), voiding cystourethrogram (VCUG). In patients presenting with urinary tract infection (UTI), surgery was delayed and antibiotic therapy was given according to urine culture results.

\section{Data}

Included stricture etiology (idiopathic, instrumentation, inflammatory), length of stricture, and preoperative maximum flow rate (pQmax).

\section{PROCEDURE}

DVIU was performed in all patients using a cold-knife cut at the 12 o'clock position. A silicon 14F Foley catheter was left in place for 5 days.

\section{Follow-up protocol}

First follow-up visit post catheter removal was scheduled at $5^{\text {th }}$ day when PUC was removed then at 1 month and then 3 monthly in the first year and every 6 monthly thereafter.

On every follow-up visit patient underwent uroflowmetry.

Success versus failure criteria

The clinical outcome was classified as a success if uroflowmetry showed Qmax $>12 \mathrm{ml} / \mathrm{s}$. 
If $\mathrm{Qmax}<12 \mathrm{ml} / \mathrm{s}$. A repeat RUG and VCUG was done.

\section{Statistical analysis}

Multivariable Cox regression analysis predicting treatment failure was done. Predictors consisted of stricture etiology (idiopathic vs nonidiopathic), stricture length, and pQmax. All statistical analyses were performed using Stata v.12.0 (StataCorp LP, College Station, TX, USA). All tests were twosided and significance was set at $\mathrm{p}<0.05$.

\section{RESULTS}

Overall, 215 patients were included. The stenosis length was less then $1 \mathrm{~cm}$ in 20 cases (9.3\%), $1-2 \mathrm{~cm}$ in 89 cases (41.3\%), $2-3 \mathrm{~cm}$ in 73 cases (33.9\%), and $3-4 \mathrm{~cm}$ in 33 cases (15.3\%). The Pre-operative maximum flow rate ( $\mathrm{ml} / \mathrm{sec}$ ) (pQmax) was less then $6 \mathrm{ml} / \mathrm{sec}$ in 32 cases (14.8\%), 6-8ml/sec in 68 cases (31.6\%) and More then $8 \mathrm{ml} / \mathrm{sec}$ in 115 cases (53.4\%). Median follow-up was 35 months (Range 12-60 months). [Table 1]

At 5-yr follow-up the failure-free survival rate was $54.4 \%$ [Table 2]. On multivariable analysis, pQmax was the only significant predictor of treatment failure $(p<0.05)$.

\section{DISCUSSION}

Our study of a homogeneous series of patients with long and strict follow-up shows: DVIU success depends on the pQmax. We report here that the DVIU success rate was $82.6 \%$ for $\mathrm{pQmax}>8 \mathrm{ml} / \mathrm{sec}$ and only $43.75 \%$ for $\mathrm{pQmax}<6 \mathrm{ml} / \mathrm{sec}$.

It is interesting to note that the time of failure recurrence after primary DVIU is homogeneously distributed over time and patients may develop stricture recurrence after many years.

The limitation of our study is the Qmax cutoff of $>12 \mathrm{ml} / \mathrm{s}$ we used as a success in follow up. Qmax normally differs by age and gender and with pathologic conditions. In cases of benign prostatic hyperplasia, urinary flow generally decreases. The Qmax lower acceptable limit is typically set to approximately $15 \mathrm{ml} / \mathrm{s}$, but an arbitrary lower limit is often considered for patients with urethral stricture disease.

Guido Barbagli et al had published similar paper in 2018 with similar findings. [9]

\section{CONCLUSIONS}

DVIU success rate for untreated bulbar urethral strictures was significantly associated with preoperative maximum flow rate. The patients with a pre- operative maximum flow lesser than $6 \mathrm{ml} / \mathrm{s}$ have a low probability of success and may be considered for alternative treatments such as open urethroplasty, especially when affected by long urethral strictures.

TABLE 1:- Characteristics of disease

\begin{tabular}{|l|l|}
\hline Etiology & Number of cases \\
\hline Idiopathic & 98 \\
\hline Instrumentation & 83 \\
\hline Inflammatory & 34 \\
\hline Length of stricture & \\
\hline Less then l cm & 20 \\
\hline $1-2 \mathrm{~cm}$ & 89 \\
\hline $2-3 \mathrm{~cm}$ & 73 \\
\hline $3-4 \mathrm{~cm}$ & 33 \\
\hline $\begin{array}{l}\text { Pre-operative maximum flow } \\
\text { rate(ml/sec) (pQmax) }\end{array}$ & \\
\hline Less then 6 ml/sec & 32 \\
\hline 6-8ml/sec & 68 \\
\hline More then 8 ml/sec & 115 \\
\hline Mean Follow up (months) & 35 \\
\hline
\end{tabular}

Table 2 :- Failure free survival rate(\%) v/s number of years post urethrotomy.

\begin{tabular}{|l|l|l|}
\hline $\begin{array}{l}\text { Time post } \\
\text { urethrotomy(years) }\end{array}$ & $\begin{array}{l}\text { Number of successful } \\
\text { treated cases }\end{array}$ & Percentage \\
\hline 1 & 156 & 72.5 \\
\hline 2 & 134 & 62.3 \\
\hline 3 & 121 & 56.2 \\
\hline 4 & 117 & 54.4 \\
\hline 5 & 108 & 50.2 \\
\hline
\end{tabular}

Conflict of interest: -

None

Research involving Human Participants :-

Retrospective analysis of data.

Informed consent:-

It was taken from all the participants.

Author's Contribution

Dr Abhiyutthan Singh jadaon :- Project development, Data Collection, Manuscript writing.

Dr Chandra pal singh :- Project development, Data Collection, Manuscript writing

Dr Chitra champawat :- Data analysis

REFERENCES:-

1. Waxman SW, Morey AF. Management of urethral strictures. Lancet 2006;367:1379-80.

2. Ferguson GG, Bullock TL, Anderson RE, et al. Minimally invasive methods for bulbar urethral strictures: a survey of members of the American Urological Association. Urology 2011;78:701-7.

3. Anger JT, Buckley JC, Santucci RA, et al. Trends in stricture manage-ment among male Medicare beneficiaries: underuse of urethro- plasty? Urology 2011;77:481-6.

4. Davis E, Lee LW. Lasting results following internal urethrotomy for urethral stricture. J Urol 1948;59:935-9.

5. Al Taweel W, Seyam R. Visual internal urethrotomy for adult male urethral stricture has poor long-term results. Adv Urol 2015;2015:1-4.

6. Santucci R, Eisenberg L. Urethrotomy has a much lower success rate than previously reported. JUrol 2010; 183:1859-62.

7. Pansadoro V, Emiliozzi P. Internal urethrotomy in the management of anterior urethral strictures. J Urol 1996;156:73-5.

8. Albers P, Fichtner J, Bruhl P, et al. Long-term results of internal urethrotomy. J Urol 1996;156:1611-4.

9. Barbagli $\mathrm{G}$, et al. Focus on Internal Urethrotomy as Primary Treatment for Untreated Bulbar Urethral Strictures: Results from a Multivariable Analysis. Eur Urol Focus (2018), https://doi.org/10.1016/j.euf.2018.10.014 